\title{
EMPLOYMENT OF THE METHOD OF FACTORIZATION FOR SOLVING PROBLEMS IN NONLINEAR DYNAMICS OF MICROTUBULES
}

\author{
Miloš Đekić \\ Institute for Multidisciplinary Research, Kneza Višeslava 1, \\ 11030 Belgrade, Republic of Serbia \\ E-mail:mdjekic@ptt.rs
}

(Received August 16, 2013)

\begin{abstract}
In the present paper we rely on the so called u-model of the dynamics of microtubules. We demonstrate how a crucial differential equation can be solved using the method of factorization. It was shown that the solutions match the ones obtained using the standard procedure.
\end{abstract}

Keywords: Microtubules, factorization, kink solitons.

\section{INTRODUCTION}

Cell cytoskeleton of eukaryotes has a dynamic nature and is composed of three types of filaments: actin filaments, intermediate filaments and microtubules [1]. Microtubules are the most stiff of the three types of filaments. They are composed of heterodimers consisting of $\alpha$ and $\beta$ tubulins. The heterodimers have the same orientation all along microtubules and their $\alpha$ and $\beta$ ends are called minus (-) and plus (+) end, respectively.

A row of $\alpha \beta$ heterodimers represents a protofilament, while 11-16 protofilaments form a hollow tube (cylinder) which is a microtubule. If the number of protofilaments in microtubule is 13 they all lie parallel to the microtubule axis. Otherwise, they wind slowly helicoidally around the microtubule axis. The length of the $\alpha \beta$ heterodimer is $l=8 \mathrm{~nm}[2,3]$ and its dipole moment is $p=337$ Debye [2,3]. $\alpha$ tubulin is positively and $\beta$ tubulin is negatively charged [2].

The minus (-) end of microtubule usually starts at an organizing center of microtubules in the cell represented with the so called centrosome, usually with a pair of centrioles [4]. In the interphase cells the centrosome is approximately at the center of the cell. The other, $(+)$ end of microtubule, radiates to the periphery of the cell. An exception to that rule is a neuron, where about $40 \%$ of microtubules in their dendrites are oriented with their (-) ends towards the neuron's periphery [4].

Outer and inner diameters of a microtubule are $25 \mathrm{~nm}$ and $15 \mathrm{~nm}$, respectively. Both $\alpha$ and $\beta$ monomers bind one GTP molecule. Soon after polymerization into a microtubule the $\beta$ monomer hydrolyses its GTP molecule to GDP and phosphate $\mathrm{P}_{\mathrm{i}}$ [4]. This hydrolysis makes the microtubules unstable and prone to disintegration (which happens always from the ends, never from the walls of the microtubule). Microtubules with slowly hydrolysable analogs of GTP are much more stable [4]. Microtubules can be as long as $20 \mu \mathrm{m}$ in vivo and $3 \mathrm{~mm}$ in vitro [4]. 
In this paper we consider the so called u-model of the microtubule dynamics. A crucial partial differential equation, which describes this dynamics, can be transformed into an ordinary differential equation. It is demonstrated that the latter one can be solved using the method of factorization. Finally, we compare solutions obtained by the method of factorization with the solutions obtained by the standard procedure. Of course, the same procedure can be applied for solving similar equation, i.e. to different models explaining dynamics of microtubules.

\section{THE U-MODEL OF DYNAMICS OF MICROTUBULES}

The u-model [2] has the same form as the z-model [3] and we get the same crucial ordinary differential equation, but the corresponding coordinates $u_{n}$ and $z_{n}$ of an $\mathrm{n}$-th $\alpha \beta$ heterodimer have different meaning. In the u-model $\alpha \beta$ heterodimers rotate but $u_{n}$ is the projection of the tip of the $\mathrm{n}$-th dimer on the protofilament direction [2]. Hence, it is common to call the model as the longitudinal one. In the z-model $\alpha \beta$ heterodimers have a longitudinal degree of freedom and $z_{n}$ is the longitudinal displacement of the $\alpha \beta$ heterodimer along protofilament direction [3].

Interaction between $\alpha \beta$ heterodimers in one protofilament is much stronger than interaction between $\alpha \beta$ heterodimers belonging to neighboring protofilaments [5,6]. Influence of neighboring protofilaments is included through a potential $V\left(u_{n}\right)$ :

$V\left(u_{n}\right)=-\frac{1}{2} A u_{n}^{2}+\frac{1}{4} B u_{n}^{4}-C u_{n}$,

where $A, B$ and $C$ are positive model parameters and $C=E q$, where $E>0$ is the intrinsic electric field and $q>0$ is the surplus of charge of the $\alpha \beta$ heterodimer.

We assume that $\alpha \beta$ heterodimer has only one degree of freedom [2,3,5]. From now on we choose the label $u_{n}$. The Hamiltonian for one protofilament of the microtubule reads $[2,3,5]$ (the Hamiltonian in the z-model is the same except for $z_{n}$ playing the role of $u_{n}$ ):

$H=\sum_{n}\left[\frac{1}{2} m \dot{u}_{n}^{2}+\frac{1}{2} k\left(u_{n+1}-u_{n}\right)^{2}+V\left(u_{n}\right)\right]$,

where dot means the first derivative with respect to time, $m$ is a mass of the $\alpha \beta$ heterodimer, $k$ is an interdimer stiffness constant and $\mathrm{n}$ counts $\alpha \beta$ heterodimers in the protofilament.

Taking

$p_{n}=m \dot{u}_{n}$

the Hamiltonian becomes

$H=\sum_{n}\left[\frac{p_{n}{ }^{2}}{2 m}+\frac{1}{2} k\left(u_{n+1}-u_{n}\right)^{2}-C u_{n}-\frac{1}{2} A{u_{n}}^{2}+\frac{1}{4} B u_{n}{ }^{4}\right]$

Equations of motion of the $\alpha \beta$ heterodimers are

$\dot{u}_{n}=\frac{\partial H}{\partial p_{n}}=\frac{p_{n}}{m}, \quad \dot{p}_{n}=-\frac{\partial H}{\partial u_{n}}+\widehat{Q}_{n}^{N P}$, 
where $\widehat{Q}_{n}^{N P}$ is a nonpotential generalized force. Our nonpotential generalized force is viscous force

$\widehat{Q}_{n}^{N P}=F_{v n}=-\dot{\mu}_{n}$,

where $\gamma$ is a viscosity coefficient.

Combining (4), (5) and (6) we get:

$m \ddot{u}_{n}=k\left(u_{n+1}+u_{n-1}-2 u_{n}\right)+q E+A u_{n}-B u_{n}{ }^{3}-\mu \dot{u}_{n}$.

Now we apply the continuum approximation $u_{n}(t) \rightarrow u(x, t)$ and use a series expansion

$u_{n \pm 1} \rightarrow u_{n} \pm \frac{\partial u_{n}}{\partial x} l+\frac{1}{2} \frac{\partial^{2} u_{n}}{\partial x^{2}} l^{2}$

where $l$ is the length of the $\alpha \beta$ heterodimer. All this brings about a following partial differential equation [2]:

$m \frac{\partial^{2} u}{\partial t^{2}}-k l^{2} \frac{\partial^{2} u}{\partial x^{2}}-q E-A u+B u^{3}+\gamma \frac{\partial u}{\partial t}=0$

We are interested in the solutions of the above equation which have the form of the traveling wave $u(\xi)$, where $\xi$ is a unified dimensionless variable

$\xi=\kappa x-\omega t$,

and $\kappa$ and $\omega$ are constants. We straightforwardly obtain an ordinary differential equation

$\left(m \omega^{2}-k l^{2} \kappa^{2}\right) u^{\prime \prime}-\gamma \omega u^{\prime}-A u+B u^{3}-q E=0$,

where $u^{\prime}=\frac{d u}{d \xi}$. Introducing a dimensionless function $\psi$ defined as

$\psi=\frac{1}{\sqrt{\frac{A}{B}}} u$,

we finally get

$\alpha \psi^{\prime \prime}-\rho \psi^{\prime}-\psi+\psi^{3}-\sigma=0$,

where

$\alpha=\frac{m \omega^{2}-k l^{2} \kappa^{2}}{A}, \quad \sigma=\frac{q E}{A \sqrt{\frac{A}{B}}}, \quad \rho=\frac{\gamma \omega}{A}$

and $\psi^{\prime}=\frac{d \psi}{d \xi}$

Equation (13) is the crucial ordinary differential equation that we want to solve using the method of factorization.

The potential which describes the influence of the neighboring protofilaments is 
$V(\psi)=\frac{A^{2}}{B}\left(\frac{1}{4} \psi^{4}-\frac{1}{2} \psi^{2}-\sigma \psi\right)=\frac{A^{2}}{B} f(\psi)$,

which can be obtained according to equations (4) and (12). The values of $\psi$ for which the potential $V(\psi)$ has extreme values are the solutions of the following equation

$f^{\prime}(\psi)=\psi^{3}-\psi-\sigma=0$

Equation (16) may have 3 solutions, $\psi_{1}, \psi_{2}$ and $\psi_{3}$ and they will be of our interest in further discussions. For $\sigma<\sigma_{0}=\frac{2}{3 \sqrt{3}}$ equation (16) has 3 real solutions, and for $\sigma \geq \sigma_{0}$ only one real solution. The solutions for $\sigma<\sigma_{0}$ are [2]:

$\psi_{1}=\frac{2}{\sqrt{3}} \cos F$,

$\psi_{2}=\frac{2}{\sqrt{3}} \cos \left(F+\frac{4 \pi}{3}\right)$

$\psi_{3}=\frac{2}{\sqrt{3}} \cos \left(F+\frac{2 \pi}{3}\right)$,

where

$F=\frac{\arccos \left(\frac{\sigma}{\sigma_{0}}\right)}{3}$.

The function $f(\psi)$ is shown in Fig. 1 for two values of the parameter $\sigma$. One can see that $\psi_{1}, \psi_{2}$ and $\psi_{3}$ describe a right minimum, a maximum and a left minimum, respectively. For $\sigma=\sigma_{0}$ the left minimum and the maximum disappear, coalescing in the saddle point.

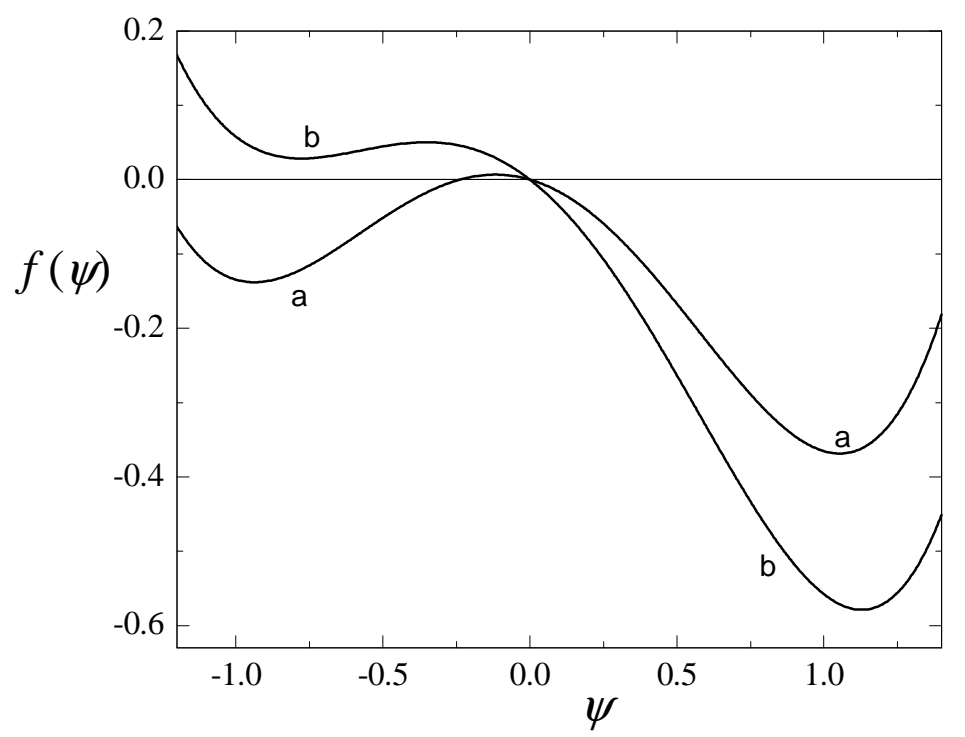

Fig. 1. The function $f(\psi)$ for: (a) $\sigma=0.3 \sigma_{0}$ and (b) $\sigma=0.8 \sigma_{0}$ 
For $\sigma \geq \sigma_{0}$ the only one real solution of equation (16) is [2]:

$$
\psi_{r}=\frac{2}{\sqrt{3}} \frac{1}{\sin \left\{2 \arctan \left[\sqrt[3]{\tan \left[\frac{1}{2} \arcsin \left(\frac{\sigma_{0}}{\sigma}\right)\right]}\right]\right\}}
$$

\section{SOLVING EQUATION (13) BY THE METHOD OF FACTORIZATION}

Our ordinary differential equation that should be solved in this Section is equation (13). Because of the free term $-\sigma$, the method of factorization cannot be immediately applied [7]. To get rid of this free term we introduce the following substitution [7]:

$\psi=\varphi+\delta$

where $\delta$ is a constant to be determined.

Inserting equation (22) into (13) we obtain:

$\alpha \varphi^{\prime \prime}-\rho \varphi^{\prime}-\left(1-3 \delta^{2}\right) \varphi+3 \delta \varphi^{2}+\varphi^{3}+\delta^{3}-\delta-\sigma=0$

which indicates

$\delta^{3}-\delta-\sigma=0$

We should notice that equation (24) is the same as (16). Hence, because of (24) equation (23) becomes

$\varphi^{\prime \prime}-\frac{\rho}{\alpha} \varphi^{\prime}-\frac{\left(1-3 \delta^{2}\right)}{\alpha} \varphi+\frac{3 \delta}{\alpha} \varphi^{2}+\frac{1}{\alpha} \varphi^{3}=0$.

We seek (25) in the form [7]:

$\left(\frac{d}{d \xi}-f_{2}\right)\left(\frac{d}{d \xi}-f_{1}\right) \varphi=0$

where $f_{1}$ and $f_{2}$ are functions of $\varphi$ to be determined. This yields:

$\frac{d^{2} \varphi}{d \xi^{2}}-\left(\frac{d f_{1}}{d \varphi} \varphi+f_{1}+f_{2}\right) \frac{d \varphi}{d \xi}+f_{1} f_{2} \varphi=0$.

Comparing equations (25) and (27) we see that $f_{1}(\varphi)$ and $f_{2}(\varphi)$ satisfy:

$\frac{d f_{1}}{d \varphi} \varphi+f_{1}+f_{2}=\frac{\rho}{\alpha}$

and

$f_{1} f_{2}=\frac{1}{\alpha}\left[-\left(1-3 \delta^{2}\right)+3 \delta \varphi+\varphi^{2}\right]=\frac{1}{\alpha}\left(\varphi-\varphi_{1}\right)\left(\varphi-\varphi_{2}\right)$,

where 
$\varphi_{1}=-\frac{3 \delta}{2}+\sqrt{1-\frac{3}{4} \delta^{2}}, \quad \varphi_{2}=-\frac{3 \delta}{2}-\sqrt{1-\frac{3}{4} \delta^{2}}$.

It was pointed out that equations (24) and (16) were equal, which means that $\delta$ can have the values given by equations (17)-(20). As $\sigma<\sigma_{0}$ has been assumed we can check that $1-\frac{3}{4} \delta^{2} \geq 0$, which means that both $\varphi_{1}$ and $\varphi_{2}$ are real.

Equation (29) indicates that we should look for the functions $f_{1}$ and $f_{2}$ in the form:

$f_{1}=\frac{a}{\alpha^{k}}\left(\varphi-\varphi_{1}\right), \quad f_{2}=\frac{1}{a \alpha^{1-k}}\left(\varphi-\varphi_{2}\right)$

where the parameters $a$ and $\alpha$ should be determined, while $k$ is the "working" parameter and, of course, the final result should not depend on it.

From (28) and (31) we easily obtain

$\left(\frac{2 a}{\alpha^{k}}+\frac{1}{a \alpha^{1-k}}\right) \varphi-\frac{a}{\alpha^{k}} \varphi_{1}-\frac{1}{a \alpha^{1-k}} \varphi_{2}=\frac{\rho}{\alpha}$

which gives the following system of equations:

$\frac{2 a}{\alpha^{k}}+\frac{1}{a \alpha^{1-k}}=0$

$-\frac{a}{\alpha^{k}} \varphi_{1}-\frac{1}{a \alpha^{1-k}} \varphi_{2}=\frac{\rho}{\alpha}$

Equation (33) can be written in a more convenient form

$2 a^{2}=-\alpha^{2 k-1}$.

It was pointed out that $k$ was a "working" parameter in a sense that the final result will not depend on it. Hence, we can safely assume an integer. According to (35) we see that

$\alpha<0$

and

$a_{1,2}= \pm \frac{|\alpha|^{k}}{\sqrt{-2 \alpha}}$

We can easily check that

$\varphi_{1}-2 \varphi_{2}>0$

for any $\sigma<\sigma_{0}$. For example, this can be seen from the graph showing the function in equation (38) as a function of $\sigma$. Of course, the expressions for $\varphi_{1}$ and $\varphi_{2}$ are given by (30), while the values for $\delta$ are given by equations (17)-(20). Hence, equations (34), (37) and (38) yield to the following important formula: 


$$
\sqrt{-\alpha}=\frac{\rho \sqrt{2}}{\varphi_{1}-2 \varphi_{2}} .
$$

Also, equations (31) and (37) bring about

$$
f_{1}= \pm \frac{1}{\sqrt{-2 \alpha}}\left(\varphi-\varphi_{1}\right)
$$

and

$$
f_{2}=\mp \sqrt{\frac{2}{-\alpha}}\left(\varphi-\varphi_{2}\right) \text {. }
$$

The functions $f_{1}$ and $f_{2}$ could have been sought in the form:

$$
f_{1}=-\frac{a}{\sqrt{-\alpha}}\left(\varphi-\varphi_{1}\right), \quad f_{2}=\frac{1}{a \sqrt{-\alpha}}\left(\varphi-\varphi_{2}\right),
$$

which was studied recently [10]. Of course, both procedures should give the same results, as was explained above.

A next step is to obtain the function $\varphi(\xi)$. Equations (26) and (40) indicate [7]:

$$
\frac{d \varphi}{d \xi}= \pm \frac{1}{\sqrt{-2 \alpha}}\left(\varphi-\varphi_{1}\right) \varphi
$$

Its direct integration brings about

$$
\log \left|\frac{\varphi-\varphi_{1}}{\varphi}\right|= \pm \frac{\varphi_{1}}{\sqrt{-2 \alpha}}\left(\xi-\xi_{0}\right)
$$

where $\xi_{0}$ is a constant of integration. A solution, having physical meaning, is

$$
\varphi(\xi)=\frac{\varphi_{1}}{1+e^{ \pm \frac{\varphi_{1}}{\sqrt{-2 \alpha} \xi}}},
$$

where $\xi_{0}=0$ is assumed. Notice that $\varphi_{1}$ is negative for $\delta=\psi_{1}$ and positive for $\delta=\psi_{2}$ and $\delta=\psi_{3}$. Expressions and signs of $\varphi_{1}$ and $\varphi_{2}$ are shown in Table 1. Obviously, the expressions for $\varphi_{1}-2 \varphi_{2}$ are crucial as can be seen from equations (39) and (45). The importance of $\varphi_{2}-2 \varphi_{1}$ will be explained later.

Table 1. Important expressions for all three values of $\delta$.

\begin{tabular}{|c|c|c|c|}
\hline$\delta$ & $\psi_{1}$ & $\psi_{2}$ & $\psi_{3}$ \\
\hline$\varphi_{1}$ & $-\sqrt{3} \cos F+\sin F<0$ & $\sqrt{3} \cos F-\sin F>0$ & $\sqrt{3} \cos F+\sin F>0$ \\
\hline$\varphi_{2}$ & $-\sqrt{3} \cos F-\sin F<0$ & $-2 \sin F<0$ & $2 \sin F>0$ \\
\hline$\varphi_{1}-2 \varphi_{2}$ & $-3 \psi_{3}>0$ & $-3 \psi_{3}>0$ & $-3 \psi_{2}>0$ \\
\hline$\varphi_{2}-2 \varphi_{1}$ & $-3 \psi_{2}>0$ & $-3 \psi_{1}<0$ & $-3 \psi_{1}<0$ \\
\hline
\end{tabular}


Finally, we can straightforwardly construct the function $\psi(\xi)$ using equations (17)-(19), (22) and (45). To understand the physical meaning of the solutions we should look for the asymptotic values $\psi( \pm \infty)$. For example, for $\delta=\psi_{1}$ and for the positive sign in (45) we obtain

$\psi(-\infty)=\psi_{1}, \quad \psi(+\infty)=\psi_{2}$.

This obviously represents a transition from the right minimum to the maximum (See Fig. 1). If we had chosen the minus sign in (45) we would have obtained $\psi(-\infty)=\psi_{2}$ and $\psi(+\infty)=\psi_{1}$, representing the same transition but in opposite direction. Following the same procedure we obtain the remaining two pairs of the asymptotic values. All this is shown in the upper half of Table 2.

Table 2. Asymptotic values of the function $\psi(\xi)$ for all three values of $\delta$.

\begin{tabular}{|c|l|l|l|l|}
\hline$\delta$ & $\psi_{1}$ & $\psi_{2}$ & $\psi_{3}$ & \\
\hline$\psi(-\infty)$ & $\psi_{1}$ & $\psi_{1}$ & $\psi_{1}$ & $f_{1}$ \\
\hline$\psi(+\infty)$ & $\psi_{2}$ & $\psi_{2}$ & $\psi_{3}$ & $f_{1}$ \\
\hline$\psi(-\infty)$ & $\psi_{1}$ & $\psi_{3}$ & $\psi_{3}$ & $f_{1}^{\prime}$ \\
\hline$\psi(+\infty)$ & $\psi_{3}$ & $\psi_{2}$ & $\psi_{2}$ & $f_{1}^{\prime}$ \\
\hline
\end{tabular}

It is obvious that (31) is not a unique choice for $f_{1}$ and $f_{2}$. Alternative possibility is

$f_{1}^{\prime}=\frac{a}{\alpha^{k}}\left(\varphi-\varphi_{2}\right), \quad f_{2}^{\prime}=\frac{1}{a \alpha^{1-k}}\left(\varphi-\varphi_{1}\right)$

Following the procedure explained above we obtain the remaining two rows in Table 2 as well as (36) and (37). Also, equation (39) becomes

$$
\sqrt{-\alpha}=\frac{\rho \sqrt{2}}{\left|\varphi_{2}-2 \varphi_{1}\right|} .
$$

The absolute value exists because the sign of $\varphi_{2}-2 \varphi_{1}$ depends on the choice of $\delta$ as shown in Table 1.

Table 2 shows that there are three pairs of the asymptotic values. However, a careful inspection of the solutions $\psi(\xi)$ shows that that there are actually three pairs of the solutions. Namely, $\delta=\psi_{1}$ and $\delta=\psi_{2}$ give the same function $\psi(\xi)$ for $f_{1}$ determined by (31) and so on. All these solutions are shown in Fig. 2 for $\rho=1$ and $\sigma=0.9 \sigma_{0}$. The corresponding asymptotic values are: $\psi_{1}=1.14, \psi_{2}=-0.42$ and $\psi_{3}=-0.72$. Hence, there are three possible solutions for equation (13). Notice that for the two solutions for which $\psi(-\infty)=\psi_{1}$ the positive sign in (45) was assumed, while the negative one was used for the remaining solution explaining transition from the left minimum to the maximum. Of course, one can say that we can change the sign in (45) and obtain three more solutions. This is correct but those new solutions would not represent any new physics. Namely, to change the sign in (45) is the same as to replace $\xi$ by $-\xi$. Obviously, kink and antikink solitons describe nonlinear dynamics of MTs. Of course, Fig. 2 was done according to equations (17)-(20), (22), (30) or Table 1, (39) and (45). 


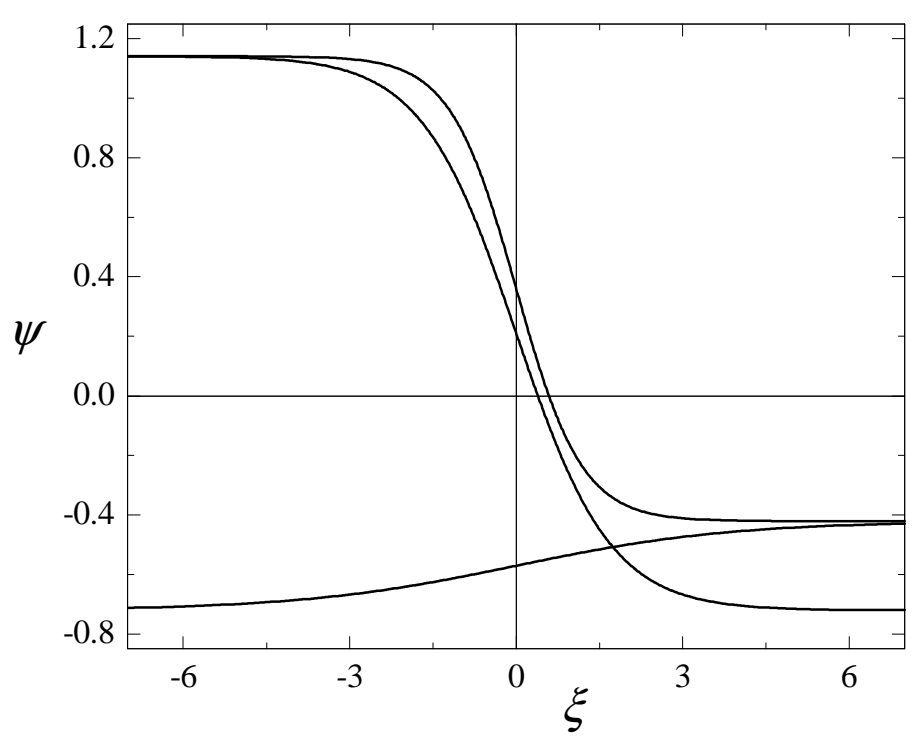

Fig. 2. The function $\psi(\xi)$ for $\rho=1$ and $\sigma=0.9 \sigma_{0}$.

\section{CONCLUSIONS AND DISCUSSIONS}

In the present paper we showed how the method of factorization can be used to solve problems in nonlinear dynamics of MTs. The procedure was demonstrated using u-model of MTs. It was shown that nonlinear MT dynamics can be represented by existence of kink and antikink solitons in MT chain. The same procedure can be applied to somewhat simpler $\varphi$ model [8].

Of course, equations like (13) can be solved using different methods. Examples could be modified extended tanh-function method [2] and the procedure based on Jacobian elliptic functions [6]. These two methods require trial functions. In this sense, the procedure used here is more general as no trial function is required.

Finally, we show that the function $\psi(\xi)$, derived in this paper, is the same as the one obtained more than 20 years ago using different procedure $[5,9,10]$, which we can call as standard method. To obtain the function $u(\xi) \equiv u$ we should use equations (12), (22) and (45). As an example we pick up the case $\delta=\psi_{3}$, for which one can easily show that

$\varphi_{1}=\psi_{1}-\psi_{3}$.

Hence, we easily obtain

$$
u=u_{3}+\frac{u_{1}-u_{3}}{1+\exp \left( \pm \sqrt{\frac{B}{A}} \frac{u_{1}-u_{3}}{\sqrt{-2 \alpha}} \xi\right)}
$$

To compare (50) with, for example, equation (22) in Ref. [9], we should comment the expression for $\alpha$. Namely, following [9] we can write

$$
k l^{2} \kappa^{2}-m \omega^{2}=\kappa^{2}\left(k l^{2}-m v^{2}\right) \equiv \kappa^{2}\left(m c_{0}^{2}-m v^{2}\right)
$$


where $c_{0}$ and $v$ are sound and solitonic velocities, respectively. It was assumed that $c_{0} \gg v$ as well as $s=x-v t$ instead of (10) above. Hence, under these circumstances, equation (50) becomes

$$
u=u_{3}+\frac{u_{1}-u_{3}}{1+\exp \left(\beta\left(u_{1}-u_{3}\right) s\right)}, \quad \beta= \pm \frac{1}{c_{0}} \sqrt{\frac{B}{2 m}},
$$

which are equations (21) and (22) in Ref. [9]. Notice that $u_{3}$ in this paper corresponds to $u_{2}$ in Gordon's notation.

It is important to point out that our approach is more general as nothing was assumed for $\alpha$. It was shown here and in Ref. [2] that $\alpha$ is negative. However, different model [8] may bring about positive $\alpha$. This means that $u(\xi)$ represents a supersonic soliton, which is not uncommon in physics [11].

Finally, one very important point deserves to be discussed. To derive equation (13) the continuum approximation was used. A key question in nonlinear biophysics is either an essentially discrete system can be considered as a continuum one. As for the MTs this question was answered recently [12]. Namely, the initial discrete equation (7) was solved numerically and the result excellently matches the one shown here, based on the continuum approximation. This proves that the continuum approximation is applicable in MT research.

\section{References:}

[1] KoRAĆ, A., Higher course in biology of cell, Biological faculty, Belgrade, (2009) (in Serbian).

[2] Zdravković, S., Kavitha, L., Satarić, M.V., Zeković, S., Petrović, J., Modified extended tanh-function method and nonlinear dynamics of microtubules", Chaos, Solitons and Fractals 45 (2012) 1378-1386.

[3] ZdRAVković, S., SATARić, M.V., Zeković, S., Nonlinear dynamics of microtubules - a longitudinal model, Europhys. Lett. 102 (2013) 38002.

[4] Pollard, T.D., Earnshaw, W.C., Lippincot-Schwartz, J., Cell Biology, 2nd edition, Saunders, Elsevier (2008).

[5] SATARIĆ, M.V., TuszYnSki, J.A., ŽAKula, R.B., Kinklike excitations as an energy transfer mechanism in microtubules, Phys. Rev. E 48 (1993) 583-597.

[6] Zeković, S., Zdravković, S., Kavitha, L., MuniYapPan, A., Employment of Jakobian elliptic functions for solving problems in nonlinear dynamics of microtubules, Chin. Phys. B 23 (2014) 020504.

[7] Cornejo-Perez, O., Negro, J., Nieto, L.M., Rosu, H.C., Traveling wave solutions for Korteweg-deVries-Burgers equations through factorizations, Found. Phys. 36 (2006) 1587-1599.

[8] Zdravković, S., Satarić, M.V., Maluckov, A., Balaž, A., A nonlinear model of the dynamics of radial dislocations in microtubules, Appl. Math. Comput. 237 (2014) 227-237

[9] GORDON A., Nonlinear mechanism for proton transfer in hydrogen-bonded solids, Physica 146B (1987) 373-378.

[10] Collins, M.A., Blumen, A., Currie, J.F., Ross, J., Dynamics of domain walls in ferrodistortive materials. I Theory, Phys. Rev. B 19 (1979) 3630-3644

[11] Davydov, A.S., Solitons in Molecular Systems, Mathematics and its Applications, D. Reidel Publishing Company, Dordrecht (1985).

[12] Zdravković, S., Maluckov, A., Đekić, M., Kuzmanović, S., Satarić, M.V., Are microtubules discrete or continuum systems?, To be published in Appl. Math. Comput. (DOI: 10.1016/j.amc.2014.05.068). 